\title{
A Novel Score Combining Magnetic Resonance Spectroscopy Parameters and Systemic Immune- inflammation Index Improves Prognosis Prediction in Non-small-cell Lung Cancer With Brain Metastases
}

\section{Dong Guo}

Hebei Medical University https://orcid.org/0000-0001-8067-5531

Jiafeng Liu

Rizhao People's Hospital

\section{Yanping Li}

Sunshine Hospital

\section{Qingqing Chen}

Nanjing Medical University Affiliated Suzhou Hospital: Suzhou Municipal Hospital

Yunzheng Zhao

Sunshine Hospital

Xinwei Guo

Yangzhou University

Shengjun Ji

Nanjing Medical University Affiliated Suzhou Hospital: Suzhou Municipal Hospital

Shuchai Zhu (D3562069484@qq.com)

Hebei Medical University Fourth Affiliated Hospital and Hebei Provincial Tumor Hospital

\section{Research Article}

Keywords: NSCLC, brain metastases, SII, MRS, prognosis

Posted Date: September 15th, 2021

DOI: https://doi.org/10.21203/rs.3.rs-874966/v1

License: (c) (i) This work is licensed under a Creative Commons Attribution 4.0 International License. Read Full License 


\section{Abstract}

Background: The aim of this study was to evaluate the significance of combination of the magnetic resonance spectroscopy (MRS) parameters and systemic immune-inflammation (SII) in patients with brain metastases (BM) from non-small-cell lung cancer (NSCLC) treated with stereotactic radiotherapy.

Methods: 118 NSCLC patients with BM who treated with stereotactic radiotherapy were retrospectively enrolled into this study. All patients underwent MRS and blood samples test for SIl analysis before the initiation of stereotactic radiotherapy. The correlation between the parameters of MRS and SIl level were assessed using the spearman correlation coefficient. The cut-off values for the parameters of MRS, SII and clinical laboratory variables were defined by the receiver operating characteristic (ROC) curve analysis to quantify these predictive value. The prognostic factors of overall survival (OS) and progression-free survival (PFS) curves were assessed using Kaplan-Meier and Cox proportional hazards models.

Results: The median follow-up time was 25 months (range, 12-49months). The optimal cutoff point for the cho/cr and SII were 1.50 and 480, respectively. The cho/cr was negatively correlated with SII (rs = $0.164, P=0.075)$, but there was a trend. C-SII score was established by combining cho/cr and SII. Patients with both an elevated cho/cr (>1.50) and an elevated SII (>480) were given a C-SII score of 2 , and patients with one or neither were given a C-SII score of 1 or 0 , respectively. Kaplan-Meier analysis showed that the C-SII score of 2 was significantly linked with poor OS and PFS $(P<0.001, P<0.001)$. In the Cox proportional hazards model, the C-SIl score independently predicted OS [hazard ratio (HR), 1.749; $95 \% \mathrm{Cl}, 1.176-2.601 ; P=0.006]$ and PFS $(\mathrm{HR}, 2.472 ; 95 \% \mathrm{Cl}, 1.624-3.763 ; P<0.001)$.

Conclusion: C-SII score was more accurate for predicting the clinical outcomes of NSCLC patients with BM who undergo stereotactic radiotherapy. The C-SIl score, which was superior to either score alone, which could be used to identify for BM from NSCLC patients with poor outcomes.

\section{Background}

Lung cancer is the most common cancer and the leading cause of cancer-related mortality in China. ${ }^{1}$ Nonsmall-cell lung cancer (NSCLC) accounts for approximately $85 \%$ of all lung cancer cases and NSCLC has a high predilection to metastasize to brain. ${ }^{2}$ Brain metastasis (BM) occurs in approximately $20-40 \%$ of patients with NSCLC, and that has become an important factor affecting the clinical outcomes of patients despite active treatments. ${ }^{3,4}$ Radiation therapy include stereotactic radiotherapy and whole brain radiation treatment. Stereotactic radiotherapy has shown advantages over whole-brain radiotherapy as the effective treatment of choice for BM patients because of excellent local control and minimal

toxicity. ${ }^{5-9}$ However, median survival time is $<12$ months and a low quality of life in patients with BM, which poses a major challenge for NSCLC patients with BM. ${ }^{10}$ Reliable prognostic indicators must be identified to look for a better treatment choice and assess patient prognosis. 
Abnormal metabolism in tumors tissue has been reached consensus and this study of metabolomics continues to provide new insight into biological behavior of the tumor. ${ }^{11}$ Advanced magnetic resonance spectroscopy (MRS) technique, can apply to various clinical questions and evaluate cellular metabolism biochemical composition. MRS technique provides the principal metabolite biochemical composition such as $\mathrm{N}$-acetyl-aspartate (NAA), choline (cho) and creatine (cr). The cho reflects synthesis and metabolism cell membranes and turnover of the cell membrane during breakdown. ${ }^{12} \mathrm{Cr}$ reflects the energy metabolism of the brain tissue cell, which are constant in normal brain tissue. ${ }^{13}$ NAA, a brain tissue neuronal marker, is reduced in the development of brain tumors. ${ }^{13}$ MRS parameters have widely been shown to be capable of identifying the biology of tumor metabolism, and can monitor the treatment effect or tumor progression. ${ }^{14,15}$ Our primary study have shown that cho/cr has been independently verified as useful predictors in the prognosis of NSCLC patients. ${ }^{16}$ However, the occurrence of tumor is a comprehensive result of various systemic disorders, and its characteristics cannot be accurately predicted simply from one aspect. Cancer-related inflammation is an necessary component of the tumor microenvironment, and inflammatory cells may play a critical role in the development of malignancies. ${ }^{17}$ Furthermore, systemic immune-inflammation involves metabolic biochemical mechanisms where cancer cells express immune-inflammatory cytokines, potentially reflecting the biological activity of tumor cells. These theories continue to enhance our understanding of tumors and inflammation. In the clinics, systemic immune-inflammation index (SII), derived from neutrophil $(N)$, lymphocyte $(L)$, and platelet $(P)$ in peripheral blood, has been deeply investigated in many malignancies. SII has become a reliable biomarker to reflect the immune and inflammation status of host and has been used as a prognostic index in multiple malignant cancers, including NSCLC. ${ }^{18-22}$ MRS represent the biological activity of cellular metabolism, while SII reflects immune-inflammation status, whereas both have been independently verified as useful predictors in the prognosis of NSCLC patients. However, the clinical data focusing on the predictive value of combination of the MRS and SII for BM in NSCLC patients treated with stereotactic radiotherapy remains unknown.

This study was performed to explore the prognostic value of C-SII score for BM in NSCLC patients treated with stereotactic radiotherapy and aimed to provide appropriate and individualized therapy in clinical treatment.

\section{Patients And Methods}

\section{Study design}

Between January 2014 and December 2017, the 118 patients with histologically confirmed NSCLC receiving stereotactic radiotherapy at Rizhao Center Hospital were retrospectively reviewed. The clinical pathological characteristics of age, gender, Karnofsky performance score, patient histology, number of BMs and TNM stage were collected for patients by the medical records. The MRS metabolism composition and immune-inflammatory parameters were obtained from the peripheral blood and from MRS, respectively. The inclusion criteria were: (i) Age > 18 years old; (ii) biopsy histological confirmation 
of NSCLC; (iii) Eastern Cooperative Oncology Group (ECOG) ranged from 0 to 2 scores; (iv) MRS imaging was obtained before treatment and no contraindications; (v) the number of $\mathrm{BM} \leq 3$; and (vi) no hematological disorders. The specific patient flow chart is shown in Fig. 1.

\section{Radiation treatment}

Enhanced computed tomography (CT) scans (Philips Brilliance Big Bore $\mathrm{CT}$ ) imaging were acquired and fused with a T1-weighted post-gadolinium MRI scan within 7 days of CT localization. The gross tumor volume (GTV) is accurately delineated by the identification of fusion images on the planning system, or 18Ffluorodeoxyglucose (FDG) positron emission tomography/computed tomography (PET/CT). At the same time, we have mapped out the critical organ structures (OAR), including the brain stem, left eye, right eye, left optic nerve, right optic nerve, lens and optic chiasm. The range of stereotactic radiotherapy doses used was 40-60 Gy. All radiation plans were created with arcs of 6-MV photons. (Fig. 2) Patients were given corresponding dehydration therapy to reduce brain edema during treatment.

\section{MRS analysis}

The brain scan sequence (MRI plain scanning, enhanced MRI scan and MRS scan ) was performed by 3.0 T clinical scanner magnetic resonance machine (Philips Healthcare, USA). We used point-resolved spectroscopic (PRESS) to perform multivoxel MRS sequence scanning and determined the region of interest (ROI) by three clinicians and radiologists. Normal brain tissue at the opposite side of the tumor lesion was selected as the reference area. ${ }^{1} \mathrm{H}$-MRS scanning parameter: TR:1500 ms, TE:135 ms, FOV: $230 \mathrm{~mm} \times 230 \mathrm{~mm}$, slice thickness: $5 \mathrm{~mm}$, scan time: 350 seconds, voxel size: $1 \times 1 \times 1 \mathrm{~mm}^{3}$. (Fig. 3) The MRS analysis software was used to automatically measure and calculate the relative metabolite intensities of the signals. MRS evaluation indicators included cho, cr, NAA, cho/ $\mathrm{Cr}$ and cho/NAA.

\section{Peripheral blood sample analysis}

We collected peripheral venous blood from the patient within 7 days before stereotactic radiotherapy. We strictly grasp the inclusion criteria and perform related index analysis. SII was calculated using the following formula: $S I I=P^{*} N / L$, which is based on platelet $(P)$, neutrophil $(N)$, and lymphocyte $(L)$ counts. NLR was defined as the absolute neutrophil count divided by the absolute lymphocyte count. PLR was defined as the absolute platelet count divided by the absolute lymphocyte count.

\section{Definition of C-SII score}

Based on the optimal cutoff value, cho/cr and SII was split into low and high groups, respectively. In this study, we constructed a novel prognostic C-SII score system, which included both cho/cr and SII. We assigned a score of $0-2$ to the cho/cr (low and high) and the SII (low and high). Patients were classified into three subgroups: low cho/cr and low SIl group (score $=0)$, low cho/cr or low SIl group (score $=1)$, and high cho/cr and high SII group (score = 2).

\section{Statistical analysis}


The predictive value of the MRS and peripheral blood parameters was assessed by calculating the area under the ROC curve (AUC). A ROC curve was generated to obtain the cho, cr, NAA, Cho/Cr, cho/NAA, N, P, L, SII, NLR and PLR cut-off values. Spearman coefficient test was performed to explore correlations between cho/cr and SII. Survival curves were analyzed to assess the survival time distribution by the Kaplan-Meier method and compared using the log-rank test to test the significance of OS and PFS among the different prognostic groups. Univariate and multivariate analysis logistic regression analyzes were used to determine independent prognostic factors. Two-sided $P$ values $<0.05$ were considered statistically significant. All analyses were conducted by using SPSS v19.0 software (SPSS, Inc., Chicago, IL, USA) and GraphPad Prism 5 software (GraphPad, San Diego, CA, USA).

\section{Results}

\section{Clinicopathological characteristics of patients}

As shown in Table 1, a total of 118 patients were enrolled in this retrospective study. Ages ranged from 52 to 71 years (median, 59 years). Most patients (54.2\%) were female and the majority of them $(74.6 \%)$ was adenocarcinoma. The findings showed $72(61.0 \%)$ with 1 brain metastases and 88(74.6) patients with neurologic symptoms. Regarding staging, 75 patients (63.6\%) had T1-T2 stage and there were $(31.4 \%)$ patients with N2 and N3 stage tumors. After a median follow up of 25 months (range 15-49 months), 69 of the $118(58.5 \%)$ patients suffered recurrence and metastasis, and $66(55.9 \%)$ patients died. 
Table 1

The clinical characteristics of all NSCLC patients with BM.

\begin{tabular}{|c|c|}
\hline Variables & Values \\
\hline \multicolumn{2}{|l|}{ Gender } \\
\hline Male & $54(45.8)$ \\
\hline Female & $64(54.2)$ \\
\hline \multicolumn{2}{|c|}{ Age (years) } \\
\hline$<60$ & $58(49.2)$ \\
\hline$\geq 60$ & $60(50.8)$ \\
\hline \multicolumn{2}{|l|}{ Smoke } \\
\hline No & $53(44.9)$ \\
\hline Yes & $65(55.1)$ \\
\hline \multicolumn{2}{|l|}{ KPS } \\
\hline $90-100$ & $55(46.6)$ \\
\hline $70-80$ & $63(53.4)$ \\
\hline \multicolumn{2}{|c|}{ Patient histology } \\
\hline SCC & $30(25.4)$ \\
\hline$A D$ & $88(74.6)$ \\
\hline \multicolumn{2}{|c|}{ Number of BMs } \\
\hline 1 & $72(61.0)$ \\
\hline $2-3$ & $46(39.0)$ \\
\hline \multicolumn{2}{|c|}{ Maximum diameter } \\
\hline$<2 \mathrm{~cm}$ & $62(52.5)$ \\
\hline$\geq 2 \mathrm{~cm}$ & $56(47.5)$ \\
\hline \multicolumn{2}{|c|}{ Neurologic symptoms } \\
\hline No & $58(49.2)$ \\
\hline Yes & $60(50.8)$ \\
\hline \multicolumn{2}{|c|}{ Tumor stage } \\
\hline T1-T2 & $75(63.6)$ \\
\hline T3-T4 & $43(36.4)$ \\
\hline
\end{tabular}




\begin{tabular}{|lc|}
\hline Variables & Values \\
\hline Node stage & \\
N0-N1 & $81(68.6)$ \\
N2-N3 & $37(31.4)$ \\
\hline TNM stage & $61(51.7)$ \\
I & $57(48.3)$ \\
II-III & \\
CEA & $70(59.3)$ \\
Normal & $48(40.7)$ \\
Elevated & \\
\hline Abbreviations: KPS, Karnofsky performance score; SCC, squamous cell carcinoma; AD, \\
adenocarcinoma; BM, brain metastases; CEA, carcinoembryonic antigen. \\
\hline
\end{tabular}

\section{Cho/Cr and SII}

With the Youden's J-statistics establishing 1.50 (sensitivity, 78.3\%; specificity, 61.2\%) as optimal cutoff values for cho/cr to predict PFS, the ROC analysis calculated the area under the curve (AUC) as 0.737. The same analysis showed that the optimal cutoff value was 480 (sensitivity, 69.6\%; specificity, 75.5\%) for SII with the largest AUC of 0.712. (Fig. 4) The cho/cr and SII level of pretreatment were $1.60(0.83-$ 5.68 ) and 481.71 (280.29-978.81), respectively. Table 2 presents the results of quantitative parameters of MRS and immune-inflammatory. We observed a negative correlation between cho/cr and SII, but there was a positive trend $(P=0.075$; Spearman's correlation coefficient, rs $=0.164)$. (Fig. 5$)$ 
Table 2

A summary of MRS metabolic characteristics and inflammation index.

Median (range)

\section{Signal}

Cho

$\mathrm{Cr}$

NAA

$\mathrm{Cho} / \mathrm{Cr}$

Cho/NAA

Peripheral blood inflammation index

\begin{tabular}{ll} 
Neutrophil & $4.91(2.36-9.88)$ \\
Lymphocyte & $1.93(0.82-3.57)$ \\
Platelet & $188(102-402)$ \\
SII & $481.71(280.29-978.81)$ \\
NLR & $2.59(1.37-5.97)$ \\
\hline PLR & $99.21(38.98-168.00)$
\end{tabular}

Abbreviations: Cho, choline; Cr, creatine; NAA, N-acetyl-aspartate; NLR: Neutrophil-to-lymphocyte ratio; PLR: Platelet-to-lymphocyte ratio; SIl: Systemic immune-inflammation index.

\section{Survival analysis}

After median 25 months' follow-up, OS (median OS 23 vs. 18 months) and PFS (median PFS 13 vs. 9 months) in the low cho/cr group as compared with the high cho/cr were significantly prolonged. In particular, patients with high SIl showed a shorter OS (median OS 18 vs. 20 months) and PFS (median PFS 9 vs. 11.5 months). (Fig. 6 ) The median OS in patients with C-SIl score 2 was significantly lower than the OS in patients with C-SII score 1 and C-SII score 0 (18 vs. 18 vs. 23 months; $P<0.001)$ (Fig. 7A). The median PFSs were 13, 9, and 9 months for patients with C-SIl score 0,1 , and 2 , respectively $(P<$ 0.001) (Fig. 7B).

Univariate and multivariate Cox regression analyses were performed to explore the factors affecting survival for in this study. The univariate analysis revealed that age $(P=0.022)$, smoke $(P=0.023)$ and KPS $(P=0.003)$ were significantly associated with OS, KPS $(P=0.014)$ and patient histology $(P=0.003)$ were significantly associated with PFS. In terms of MRS and peripheral blood parameters, PLR $(P=0.033$, $P=0.006), \operatorname{SI}(P=0.001, P=0.002)$ and $\operatorname{Cho} / \operatorname{Cr}(P=0.007, P<0.001)$ were positively associated with $0 \mathrm{~S}$ 
and PFS. We found no significant association of survival with number of BMs, maximum diameter, neurologic symptoms, and TNM stage. (Table 3,4) 
Table 3

Univariate analysis of OS and PFS based on clinical-pathological data

\begin{tabular}{|c|c|c|c|c|c|c|}
\hline Variables & OS & & & PFS & & \\
\hline & HR & $95 \% \mathrm{Cl}$ & P-value & HR & $95 \% \mathrm{Cl}$ & P-value \\
\hline \multicolumn{7}{|l|}{ Gender } \\
\hline Male & Reference & & & Reference & & \\
\hline Female & 0.911 & $0.560-1.482$ & 0.707 & 0.929 & $0.577-1.495$ & 0.761 \\
\hline \multicolumn{7}{|c|}{ Age (years) } \\
\hline$<60$ & Reference & & & Reference & & \\
\hline$\geq 60$ & 1.791 & $1.088-2.946$ & 0.022 & 1.476 & $0.909-2.386$ & 0.115 \\
\hline \multicolumn{7}{|l|}{ Smoke } \\
\hline No & Reference & & & Reference & & \\
\hline Yes & 1.847 & $1.089-3.133$ & 0.023 & 1.536 & $0.937-2.517$ & 0.089 \\
\hline \multicolumn{7}{|l|}{ KPS } \\
\hline $90-100$ & Reference & & & Reference & & \\
\hline $70-80$ & 2.206 & $1.298-3.750$ & 0.003 & 1.874 & $1.133-3.099$ & 0.014 \\
\hline \multicolumn{7}{|c|}{ Patient histology } \\
\hline SCC & Reference & & & Reference & & \\
\hline$A D$ & 1.294 & $0.717-2.337$ & 0.392 & 2.797 & $1.418-5.519$ & 0.003 \\
\hline \multicolumn{7}{|c|}{ Number of BMs } \\
\hline 1 & Reference & & & Reference & & \\
\hline $2-3$ & 0.828 & $0.501-1.371$ & 0.464 & 0.879 & $0.536-1.441$ & 0.608 \\
\hline \multicolumn{7}{|c|}{ Maximum diameter } \\
\hline$<2 \mathrm{~cm}$ & Reference & & & Reference & & \\
\hline$\geq 2 \mathrm{~cm}$ & 0.976 & $0.602-1.582$ & 0.920 & 1.264 & $0.784-2.039$ & 0.336 \\
\hline \multicolumn{7}{|c|}{ Neurologic symptoms } \\
\hline No & Reference & & & Reference & & \\
\hline Yes & 0.733 & $0.449-1.196$ & 0.213 & 0.868 & $0.538-1.400$ & 0.562 \\
\hline \multicolumn{7}{|c|}{ Tumor stage } \\
\hline T1-T2 & Reference & & & Reference & & \\
\hline
\end{tabular}




\begin{tabular}{|c|c|c|c|c|c|c|}
\hline T3-T4 & 0.604 & $0.349-1.047$ & 0.072 & 0.834 & $0.501-1.388$ & 0.484 \\
\hline \multicolumn{7}{|c|}{ Node stage } \\
\hline N0-N1 & Reference & & & Reference & & \\
\hline N2-N3 & 0.782 & $0.449-1.362$ & 0.385 & 1.077 & $0.642-1.806$ & 0.778 \\
\hline \multicolumn{7}{|c|}{ TNM stage } \\
\hline I & Reference & & & Reference & & \\
\hline$\| I-I I \mid$ & 1.048 & $0.639-1.718$ & 0.852 & 1.136 & $0.704-1.834$ & 0.602 \\
\hline \multicolumn{7}{|l|}{ CEA } \\
\hline Normal & Reference & & & Reference & & \\
\hline Elevated & 0.879 & $0.535-1.444$ & 0.611 & 1.158 & $0.717-1.870$ & 0.548 \\
\hline
\end{tabular}


Table 4

Univariate analysis of OS and PFS based on MRS metabolic characteristics and inflammation index.

\begin{tabular}{|c|c|c|c|c|c|c|}
\hline Variables & os & & & PFS & & \\
\hline & HR & $95 \% \mathrm{Cl}$ & P-value & HR & $95 \% \mathrm{Cl}$ & P-value \\
\hline \multicolumn{7}{|l|}{ Cho } \\
\hline$\leq 2.50$ & Reference & & & Reference & & \\
\hline$>2.50$ & 1.223 & $0.696-2.150$ & 0.484 & 1.551 & $0.902-2.665$ & 0.113 \\
\hline \multicolumn{7}{|l|}{$\mathrm{Cr}$} \\
\hline$\leq 0.50$ & Reference & & & Reference & & \\
\hline$>0.50$ & 0.641 & $0.155-2.648$ & 0.539 & 0.501 & $0.121-2.074$ & 0.341 \\
\hline \multicolumn{7}{|l|}{ NAA } \\
\hline$\leq 1.50$ & Reference & & & Reference & & \\
\hline$>1.50$ & 0.896 & $0.325-2.472$ & 0.832 & 1.188 & $0.477-2.960$ & 0.712 \\
\hline \multicolumn{7}{|l|}{ Cho/Cr } \\
\hline$\leq 1.50$ & Reference & & & Reference & & \\
\hline$>1.50$ & 2.140 & $1.230-3.723$ & 0.007 & 2.863 & $1.586-5.169$ & $\nabla 0.001$ \\
\hline \multicolumn{7}{|l|}{ Cho/NAA } \\
\hline$\leq 3.50$ & Reference & & & Reference & & \\
\hline$>3.50$ & 1.485 & $0.903-2.442$ & 0.119 & 1.619 & $0.995-2.633$ & 0.052 \\
\hline \multicolumn{7}{|l|}{ Neutrophil } \\
\hline$\leq 6.13$ & Reference & & & & & \\
\hline$>6.13$ & 1.682 & $0.993-2.849$ & 0.053 & 1.581 & $0.935-2.671$ & 0.087 \\
\hline \multicolumn{7}{|c|}{ Lymphocyte } \\
\hline$\leq 2.70$ & Reference & & & Reference & & \\
\hline$>2.70$ & 1.507 & $0.717-3.170$ & 0.280 & 1.441 & $0.727-2.858$ & 0.296 \\
\hline \multicolumn{7}{|l|}{ Platelet } \\
\hline$\leq 169$ & Reference & & & Reference & & \\
\hline >169 & 1.336 & $0.794-2.246$ & 0.275 & 1.361 & $0.812-2.280$ & 0.242 \\
\hline \multicolumn{7}{|l|}{ SII } \\
\hline$\leq 480$ & Reference & & & Reference & & \\
\hline
\end{tabular}




\begin{tabular}{|c|c|c|c|c|c|c|}
\hline$>480$ & 2.513 & $1.445-4.370$ & 0.001 & 2.297 & $1.370-3.851$ & 0.002 \\
\hline \multicolumn{7}{|l|}{ NLR } \\
\hline$\leq 2.50$ & Reference & & & Reference & & \\
\hline$>2.50$ & 1.296 & $0.769-2.187$ & 0.330 & 1.182 & $0.724-1.929$ & 0.503 \\
\hline \multicolumn{7}{|l|}{ PLR } \\
\hline$\leq 91.50$ & Reference & & & Reference & & \\
\hline$>91.50$ & 1.854 & $1.053-3.265$ & 0.033 & 2.211 & $1.261-3.876$ & 0.006 \\
\hline
\end{tabular}

To verify the predictive value of C-SII score in NSCLC with BM, we performed multivariate Cox proportional hazards regression analysis include age, smoke, KPS, patient histology and C-SII score. The C-SII score were independent prognostic factors for OS (HR, 1.749; 95\% $\mathrm{Cl}, 1.176-2.601 ; P=0.006)$ and PFS (HR, 2.472; 95\% Cl, 1.624-3.763; $P<0.001$ ). (Table 5) 
Table 5

Multivariate Cox regression analysis of OS and PFS

\begin{tabular}{|c|c|c|c|c|c|c|}
\hline Variables & OS & & & PFS & & \\
\hline & HR & $95 \% \mathrm{Cl}$ & P-value & HR & $95 \% \mathrm{Cl}$ & P-value \\
\hline \multicolumn{7}{|c|}{ Age (years) } \\
\hline$<60$ & Reference & & & & & \\
\hline$\geq 60$ & 1.749 & $1.028-2.978$ & 0.039 & & & \\
\hline \multicolumn{7}{|l|}{ Smoke } \\
\hline No & Reference & & & & & \\
\hline Yes & 1.106 & $0.618-1.980$ & 0.735 & & & \\
\hline \multicolumn{7}{|l|}{ KPS } \\
\hline $90-100$ & Reference & & & Reference & & \\
\hline $70-80$ & 1.515 & $0.848-2.706$ & 0.161 & 1.263 & $0.746-2.139$ & 0.385 \\
\hline \multicolumn{7}{|c|}{ Patient histology } \\
\hline ScC & & & & Reference & & \\
\hline$A D$ & & & & 4.217 & $1.657-10.736$ & 0.003 \\
\hline \multicolumn{7}{|l|}{ PLR } \\
\hline$\leq 91.50$ & Reference & & & Reference & & \\
\hline$>91.50$ & 1.209 & $0.654-2.237$ & 0.545 & 0.556 & $0.242-1.273$ & 0.165 \\
\hline \multicolumn{7}{|l|}{ C-SII score } \\
\hline 0 & Reference & & & Reference & & \\
\hline $1-2$ & 1.749 & $1.176-2.601$ & 0.006 & 2.472 & $1.624-3.763$ & $\otimes 0.001$ \\
\hline
\end{tabular}

\section{Discussion}

Reliable prognostic prediction score system is crucial in risk stratification for patients and adjusting appropriate treatment strategy for NSCLC with BM. In the present study, we investigated the utility of cho/cr and SII and C-SII score on prognosis in NSCLC patients. The results showed that C-SII score is independent predictors of OS and PFS among NSCLC patients with BM. 
In recent years, stereotactic radiotherapy is used to treat limited numbers of $B M$, since this therapy is less invasive than drug and surgical resection with better local control. ${ }^{23,24}$ Therefore, the use of stereotactic radiotherapy is recommended to further control BM risk. ${ }^{25}$ At present, various blood indicators have been evaluated the prognosis role of NSCLC patients with BM, such as Neuron-specific enolase (NSE), ${ }^{26}$ Carcinoembryonic antigen (CEA) ${ }^{27}$ and Lung-molGPA. ${ }^{28}$ However, there are no more reliable predictors that can reflect different tumor biological behavior. Hence, searching for accurate prognostic factors is of great clinical application value.

The occurrence of tumor is often accompanied by changes in metabolism biochemical composition. MRS is a noninvasive and sensitive imaging method that allows researchers to measure and visualize metabolism biochemical information from brain tumor tissues. ${ }^{29}$ Increasing evidence have indicated that MRS can identify tumor active region and enhance more individualized response-based treatment in High-grade Glioma. ${ }^{15}$ An ongoing effort at Tehran University of Medical Sciences has shown that MRS parameters can improve the accuracy of predictive nomograms to assess the risk of biochemical recurrence after radical prostatectomy in prostate cancer. ${ }^{30}$ More comprehensive understanding the biochemical composition changes in metabolites for tumors is more urgent. The typical MRS metabolic abnormalities of BM often include increased cho, decreased NAA and cr. Minicozzi et al observed in thirtysix head and neck cancer cases that cho/cr is significantly elevated in the group with poor response. ${ }^{14}$ Fink et al found that multi-voxel MRS cho/cr peak-area shows great advantage for distinguishing glioma recurrence. ${ }^{31}$ Negendank and colleagues conducted a co-operative study with 15 clinical research centers and confirmed that the cho was higher in glial tumors than in non-involved brain tissues. ${ }^{32}$ Dowling and coworkers revealed that cho concentrations and NAA in tumor tissue were higher than normal values cancer. ${ }^{33}$ In this study, our result reported that the cho/cr was independent relevant factors for death and progression $(P=0.006 ; P<0.001)$. Whether the cholcr were not correlated with prognosis on NSCLC with BM.

Accumulating studies have substantiated that peripheral venous blood markers can reflect the condition of the host immune inflammation status. Counts of the peripheral immune inflammatory cells, such as platelet, lymphocytes, and neutrophils have been confirmed the reliable association link between inflammatory cells and prognosis in malignant tumors. ${ }^{34-37} \mathrm{SII}$ is an integrated parameter, including platelets, neutrophils and lymphocytes and has been proved to be an independent predictor of malignant tumors. ${ }^{22,38-43}$ The value of SII in predicting clinical outcomes for cancer patients may be associated with the function of platelets, neutrophils, and lymphocytes. Platelets release growth factors and proangiogenic protein and protect tumor cells from immune response. ${ }^{44}$ Neutrophils can take part in various stages of growth and metastasis of tumors and generate immunosuppressive effects by producing and secreting cytokines, chemokines, and proteases. ${ }^{45}$ In contrast to neutrophils, lymphocytes exert important antitumor immune response and have been proved to be related to systematic immune surveillance. ${ }^{46}$ In this study, the association between SII and clinical outcomes of patients in NSCLC with BM was 
evaluated. Our results indicated that SII was significantly associated with OS and PFS $(P=0.001 ; P=$ $0.002)$.

The clinician can evaluate clinical indicators such as tumor size, degree of tumor differentiation or tumor location, but these evaluation criteria are based on individual subjective evaluation and judgment. The heterogeneity of individual tumors is largely reflected in the biological characteristics of tumors and host immune inflammatory state. Consider a problem from multiple angles and you may find new breakthroughs. Recently, many scholars have realized that combining two peripheral blood indexes can be considered useful independent prognostic markers in tumors. In the retrospective study initiated by Chen et al, their results revealed that the combination of circulating tumor cells with carcinoembryonic antigen has a better disease prediction than they were alone in NSCLC patients. ${ }^{47}$ Huang et al showed that preoperative combining neutrophil-to-lymphocyte ratio and fibrinogen concentration can be used an independent prognostic indicator for OS (HR, 1.512; 95\% Cl, 1.283-1.783; $P<0.001) .{ }^{48}$ Although Guo et $\mathrm{al}^{49}$ and Schernberg et al ${ }^{50}$ conducted related studies on PET/CT combined with blood inflammation indicators predicting prognosis and obtained positive results, the clinical data focusing on the predictive value of combination of the MRS and SII for BM in NSCLC patients treated with stereotactic radiotherapy has not been reported. We previously used MRS alone to evaluate the prognosis of patients with BM in NSCLC patients and revealed that positive cho/cr was an independent risk factor for OS $(P=0.009)$ and PFS $(P=0.006) .{ }^{16}$ The cho/cr or SII alone may are not sufficient to accurately reflect the tumor characteristics. Using C-SII scores system, may be a more accurate choice. As we have studied, our results revealed that C-SII score of 2 (cho/cr $>1.50$ and SII $>480$ ) has a poorer clinical outcomes than patients with C-SII score 1 (cho/cr $>1.50$ or SII $>480$ ) or C-SII score 0 (cho/cr $\leq 1.50$ and SII $\leq 480$ ). In the multivariate Cox regression analyses, study results demonstrated that the C-SII score independently predicted OS (HR, 1.749; 95\% Cl, 1.176-2.601; $P=0.006)$ and PFS (HR, 2.472; 95\% Cl, 1.624-3.763; $P<$ $0.001)$.

We established a C-SII score system by combining cho/cr and SII in this study, and preliminary results showed that it was an accurate and reliable system for evaluating prognosis in NSCLC patients with BM. However, only 118 NSCLC with BM patients were conducted in this study because of the available limited number of enrolled cases. In addition, incomplete clinical data and loss to follow-up were inevitable because of the long duration of this retrospective study. There is selection bias when clinicians and radiologists use MRS to determine the ROI. These limitations require further evaluation and improvements in the future study.

\section{Conclusions}

High cho/cr and high SII for NSCLC patients were significantly to have poor outcomes in present study. CSII score system may be used as a strong unfavorable survival index and assess risk stratification for NSCLC patients with BM, which suggest clinicians to adjust the treatment strategy and generalize clinical application. 


\section{Declarations}

\section{Acknowledgements}

The authors acknowledge Wang Jing for his professional statistical analysis.

\section{Authors' contributions}

Dong Guo, Jiafeng Liu, Yanping Li, Qingqing Chen, Yunzheng Zhao, Xinwei Guo, Shengjun Ji and Shuchai Zhu prepared and reviewed the manuscript. All authors contributed to the article and approved the submitted version.

\section{Funding}

This study was supported by Grant (GSWS2020067) from the Gusu Health Talent Program.

\section{Availability of data and materials}

All data included in the present study were presented in the main manuscript

\section{Consent for publication}

Informed consent was obtained from all participants for publication.

\section{Ethics approval and consent to participate}

This study was approved by the ethics committee of the Rizhao Center Hospital. Informed consent was exempted due to the retrospective nature of the study.

\section{Competing interests}

The authors declare that they have no conflict of interest

\section{References}

1. Chen W, Zheng R, Baade PD, et al. Cancer statistics in China, 2015. CA Cancer J Clin. 2016;66(2):115132.

2. Pugh TJ, Gaspar LE. Prophylactic cranial irradiation for patients with lung cancer. Clin Lung Cancer. 2007;8(6):365-368.

3. Zakaria R, Das K, Bhojak M, Radon M, Walker C, Jenkinson MD. The role of magnetic resonance imaging in the management of brain metastases: diagnosis to prognosis. Cancer Imaging. 2014;14:8.

4. Wolf A, Kvint S, Chachoua A, et al. Toward the complete control of brain metastases using surveillance screening and stereotactic radiosurgery. J Neurosurg. 2018;128(1):23-31. 
5. Ostrom QT, Wright CH, Barnholtz-Sloan JS. Brain metastases: epidemiology. Handb Clin Neurol. 2018;149:27-42.

6. Sallabanda M, Garcia-Berrocal MI, Romero J, et al. Brain metastases treated with radiosurgery or hypofractionated stereotactic radiotherapy: outcomes and predictors of survival. Clin Trans/ Oncol. 2020;22(10):1809-1817.

7. Nieder C, Mehta MP, Geinitz H, Grosu AL. Prognostic and predictive factors in patients with brain metastases from solid tumors: A review of published nomograms. Crit Rev Oncol Hematol. 2018;126:13-18.

8. Brown PD, Jaeckle K, Ballman KV, et al. Effect of Radiosurgery Alone vs Radiosurgery With Whole Brain Radiation Therapy on Cognitive Function in Patients With 1 to 3 Brain Metastases: A Randomized Clinical Trial. JAMA. 2016;316(4):401-409.

9. Yamamoto M, Serizawa T, Shuto T, et al. Stereotactic radiosurgery for patients with multiple brain metastases (JLGK0901): a multi-institutional prospective observational study. Lancet Oncol. 2014;15(4):387-395.

10. Chen AM, Jahan TM, Jablons DM, Garcia J, Larson DA. Risk of cerebral metastases and neurological death after pathological complete response to neoadjuvant therapy for locally advanced nonsmallcell lung cancer: clinical implications for the subsequent management of the brain. Cancer. 2007;109(8):1668-1675.

11. Vander Heiden MG, Cantley LC, Thompson CB. Understanding the Warburg effect: the metabolic requirements of cell proliferation. Science. 2009;324(5930):1029-1033.

12. Miller $B L$, Chang $L$, Booth $R$, et al. In vivo $1 H$ MRS choline: correlation with in vitro chemistry/histology. Life Sci. 1996;58(22):1929-1935.

13. Maheshwari SR, Fatterpekar GM, Castillo M, Mukherji SK. Proton MR spectroscopy of the brain. Semin Ultrasound CT MR. 2000;21(6):434-451.

14. Minicozzi A, Mosconi E, Cordiano C, et al. Proton magnetic resonance spectroscopy: ex vivo study to investigate its prognostic role in colorectal cancer. Biomed Pharmacother. 2013;67(7):593-597.

15. Quon H, Brunet B, Alexander A, et al. Changes in serial magnetic resonance spectroscopy predict outcome in high-grade glioma during and after postoperative radiotherapy. Anticancer Res. 2011;31(10):3559-3565.

16. Jia C, Li Z, Guo D, et al. Brain Metastases of Non-Small Cell Lung Cancer: Magnetic Resonance Spectroscopy for Clinical Outcome Assessment in Patients with Stereotactic Radiotherapy. Onco Targets Ther. 2020;13:13087-13096.

17. Grivennikov SI, Greten FR, Karin M. Immunity, inflammation, and cancer. Cell. 2010;140(6):883-899.

18. Aziz MH, Sideras K, Aziz NA, et al. The Systemic-immune-inflammation Index Independently Predicts Survival and Recurrence in Resectable Pancreatic Cancer and its Prognostic Value Depends on Bilirubin Levels: A Retrospective Multicenter Cohort Study. Ann Surg. 2019;270(1):139-146.

19. Gao Y, Zhang H, Li Y, Wang D, Ma Y, Chen Q. Preoperative increased systemic immune-inflammation index predicts poor prognosis in patients with operable non-small cell lung cancer. Clinica chimica 
acta; international journal of clinical chemistry. 2018;484:272-277.

20. Huang L, Liu S, Lei Y, et al. Systemic immune-inflammation index, thymidine phosphorylase and survival of localized gastric cancer patients after curative resection. Oncotarget. 2016;7(28):4418544193.

21. Nie D, Gong H, Mao X, Li Z. Systemic immune-inflammation index predicts prognosis in patients with epithelial ovarian cancer: A retrospective study. Gynecol Oncol. 2019;152(2):259-264.

22. Yang R, Chang Q, Meng X, Gao N, Wang W. Prognostic value of Systemic immune-inflammation index in cancer: A meta-analysis. Journal of Cancer. 2018;9(18):3295-3302.

23. O'Neill BP, Iturria NJ, Link MJ, Pollock BE, Ballman KV, O'Fallon JR. A comparison of surgical resection and stereotactic radiosurgery in the treatment of solitary brain metastases. Int $J$ Radiat Oncol Biol Phys. 2003;55(5):1169-1176.

24. Rades D, Veninga T, Hornung D, Wittkugel O, Schild SE, Gliemroth J. Single brain metastasis: wholebrain irradiation plus either radiosurgery or neurosurgical resection. Cancer. 2012;118(4):1138-1144.

25. Rades D, Blanck O, Khoa MT, et al. Validation of a Survival Score for Patients Receiving Radiosurgery or Fractionated Stereotactic Radiotherapy for 1 to 3 Brain Metastases. In Vivo. 2018;32(2):381-384.

26. Chen $Y$, Peng W, Huang $Y$, et al. [Significance of serum neuron-specific enolase before treatment in predicting brain metastases and prognosis of advanced non-small cell lung cancer]. Zhonghua zhong liu za zhi [Chinese journal of oncology]. 2015;37(7):508-511.

27. Arrieta O, Saavedra-Perez D, Kuri R, et al. Brain metastasis development and poor survival associated with carcinoembryonic antigen (CEA) level in advanced non-small cell lung cancer: a prospective analysis. BMC Cancer. 2009;9:119.

28. Sperduto PW, Yang TJ, Beal K, et al. Estimating Survival in Patients With Lung Cancer and Brain Metastases: An Update of the Graded Prognostic Assessment for Lung Cancer Using Molecular Markers (Lung-molGPA). JAMA Oncol. 2017;3(6):827-831.

29. Mclntyre DJ, Madhu B, Lee SH, Griffiths JR. Magnetic resonance spectroscopy of cancer metabolism and response to therapy. Radiat Res. 2012;177(4):398-435.

30. Parizi MK, Razi A, Alizadeh S, Kasaeian A. The Role of Magnetic Resonance Spectroscopy Imaging Parameters to Predict Early Biochemical Recurrence after Radical Prostatectomy. Prague Med Rep. 2019;120(2-3):74-83.

31. Fink JR, Carr RB, Matsusue E, et al. Comparison of 3 Tesla proton MR spectroscopy, MR perfusion and MR diffusion for distinguishing glioma recurrence from posttreatment effects. J Magn Reson Imaging. 2012;35(1):56-63.

32. Negendank WG, Sauter R, Brown TR, et al. Proton magnetic resonance spectroscopy in patients with glial tumors: a multicenter study. J Neurosurg. 1996;84(3):449-458.

33. Dowling C, Bollen AW, Noworolski SM, et al. Preoperative proton MR spectroscopic imaging of brain tumors: correlation with histopathologic analysis of resection specimens. AJNR Am J Neuroradiol. 2001;22(4):604-612. 
34. Grenader T, Nash S, Plotkin Y, et al. Derived neutrophil lymphocyte ratio may predict benefit from cisplatin in the advanced biliary cancer: the ABC-02 and BT-22 studies. Annals of oncology : official journal of the European Society for Medical Oncology. 2015;26(9):1910-1916.

35. Kao SC, Pavlakis N, Harvie R, et al. High blood neutrophil-to-lymphocyte ratio is an indicator of poor prognosis in malignant mesothelioma patients undergoing systemic therapy. Clinical cancer research : an official journal of the American Association for Cancer Research. 2010;16(23):58055813.

36. Templeton AJ, McNamara MG, Seruga B, et al. Prognostic role of neutrophil-to-lymphocyte ratio in solid tumors: a systematic review and meta-analysis. J Natl Cancer Inst. 2014;106(6):dju124.

37. Yao M, Liu Y, Jin H, et al. Prognostic value of preoperative inflammatory markers in Chinese patients with breast cancer. Onco Targets Ther. 2014;7:1743-1752.

38. Hong X, Cui B, Wang M, Yang Z, Wang L, Xu Q. Systemic Immune-inflammation Index, Based on Platelet Counts and Neutrophil-Lymphocyte Ratio, Is Useful for Predicting Prognosis in Small Cell Lung Cancer. The Tohoku journal of experimental medicine. 2015;236(4):297-304.

39. Tong YS, Tan J, Zhou XL, Song YQ, Song YJ. Systemic immune-inflammation index predicting chemoradiation resistance and poor outcome in patients with stage III non-small cell lung cancer. Journal of translational medicine. 2017;15(1):221.

40. Yucel S, Bilgin B. The prognostic values of systemic immune-inflammation index and derived neutrophil-lymphocyte ratio in EGFR-mutant advanced non-small cell lung cancer. J Oncol Pharm Pract. 2021;27(1):71-77.

41. Bilgetekin I, Basal FB. Systemic Immune-inflammation Index is the Best Prognostic Factor in Patients with Advanced Stage Adenocarcinoma of the Lung Treated with Pemetrexed. J Coll Physicians Surg Pak. 2020;30(9):933-939.

42. Shinko D, Diakos Cl, Clarke SJ, Charles KA. Cancer-Related Systemic Inflammation: The Challenges and Therapeutic Opportunities for Personalized Medicine. Clin Pharmacol Ther. 2017;102(4):599610.

43. Tomita M, Ayabe T, Maeda R, Nakamura K. Systemic Immune-inflammation Index Predicts Survival of Patients After Curative Resection for Non-small Cell Lung Cancer. In Vivo. 2018;32(3):663-667.

44. Franco AT, Corken A, Ware J. Platelets at the interface of thrombosis, inflammation, and cancer. Blood. 2015;126(5):582-588.

45. Coffelt SB, Wellenstein MD, de Visser KE. Neutrophils in cancer: neutral no more. Nat Rev Cancer. 2016;16(7):431-446.

46. Al-Shibli KI, Donnem T, Al-Saad S, Persson M, Bremnes RM, Busund LT. Prognostic effect of epithelial and stromal lymphocyte infiltration in non-small cell lung cancer. Clinical cancer research : an official journal of the American Association for Cancer Research. 2008;14(16):5220-5227.

47. Chen X, Wang X, He H, Liu Z, Hu JF, Li W. Combination of circulating tumor cells with serum carcinoembryonic antigen enhances clinical prediction of non-small cell lung cancer. PloS one. 2015;10(5): $\mathrm{e} 0126276$. 
48. Huang W, Wang S, Zhang H, Zhang B, Wang C. Prognostic significance of combined fibrinogen concentration and neutrophil-to-lymphocyte ratio in patients with resectable non-small cell lung cancer. Cancer Biol Med. 2018;15(1):88-96.

49. Guo D, Jin F, Jing W, et al. Incorporation of the SUVmax Measured From FDG PET and Neutrophil-tolymphocyte Ratio Improves Prediction of Clinical Outcomes in Patients With Locally Advanced Nonsmall-cell Lung Cancer. Clin Lung Cancer. 2019;20(6):412-419.

50. Schernberg A, Reuze S, Orlhac F, et al. A score combining baseline neutrophilia and primary tumor SUVpeak measured from FDG PET is associated with outcome in locally advanced cervical cancer. Eur J Nucl Med Mol Imaging. 2018;45(2):187-195.

\section{Figures}

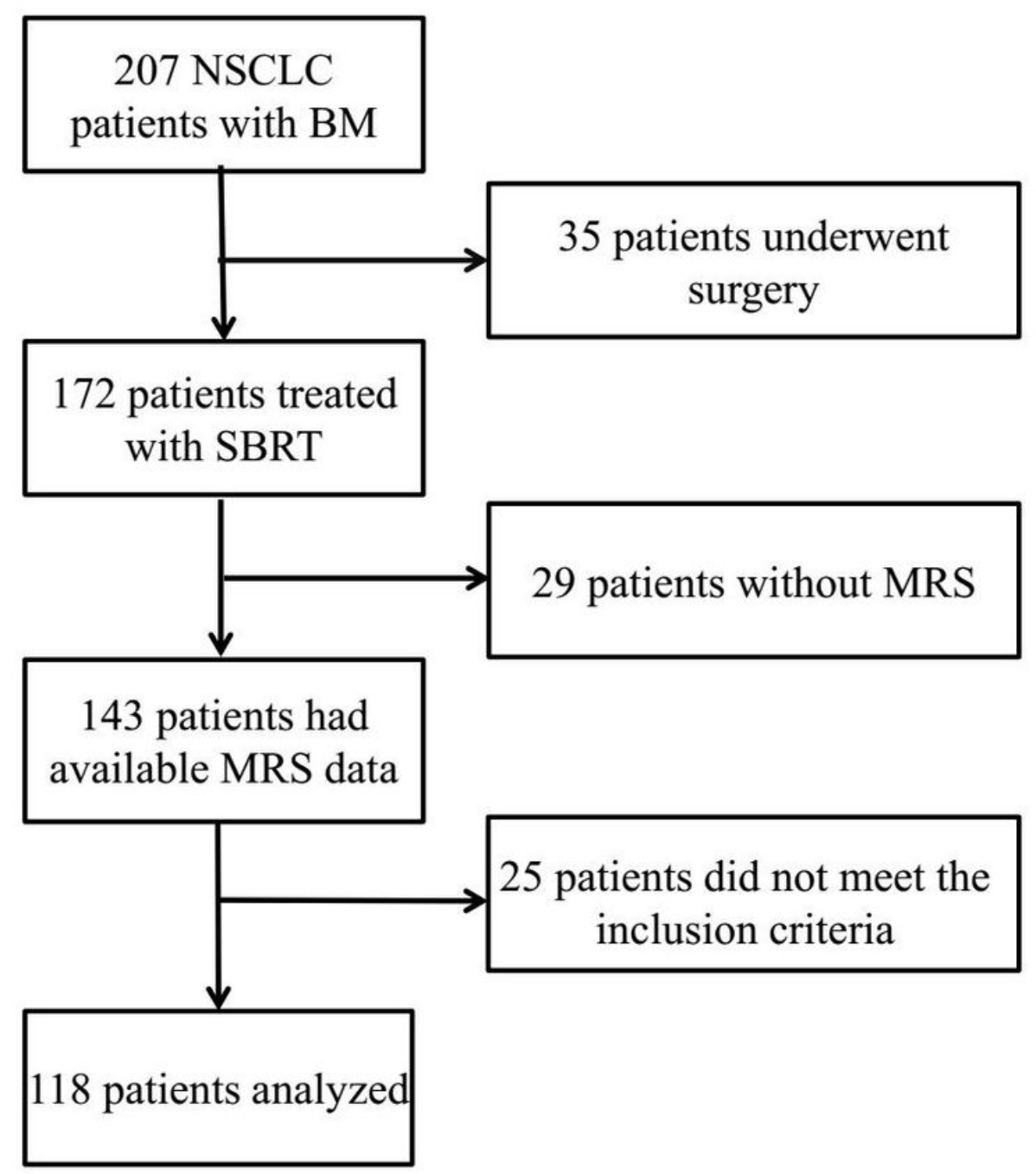

Figure 1

Patients screening process and results. 


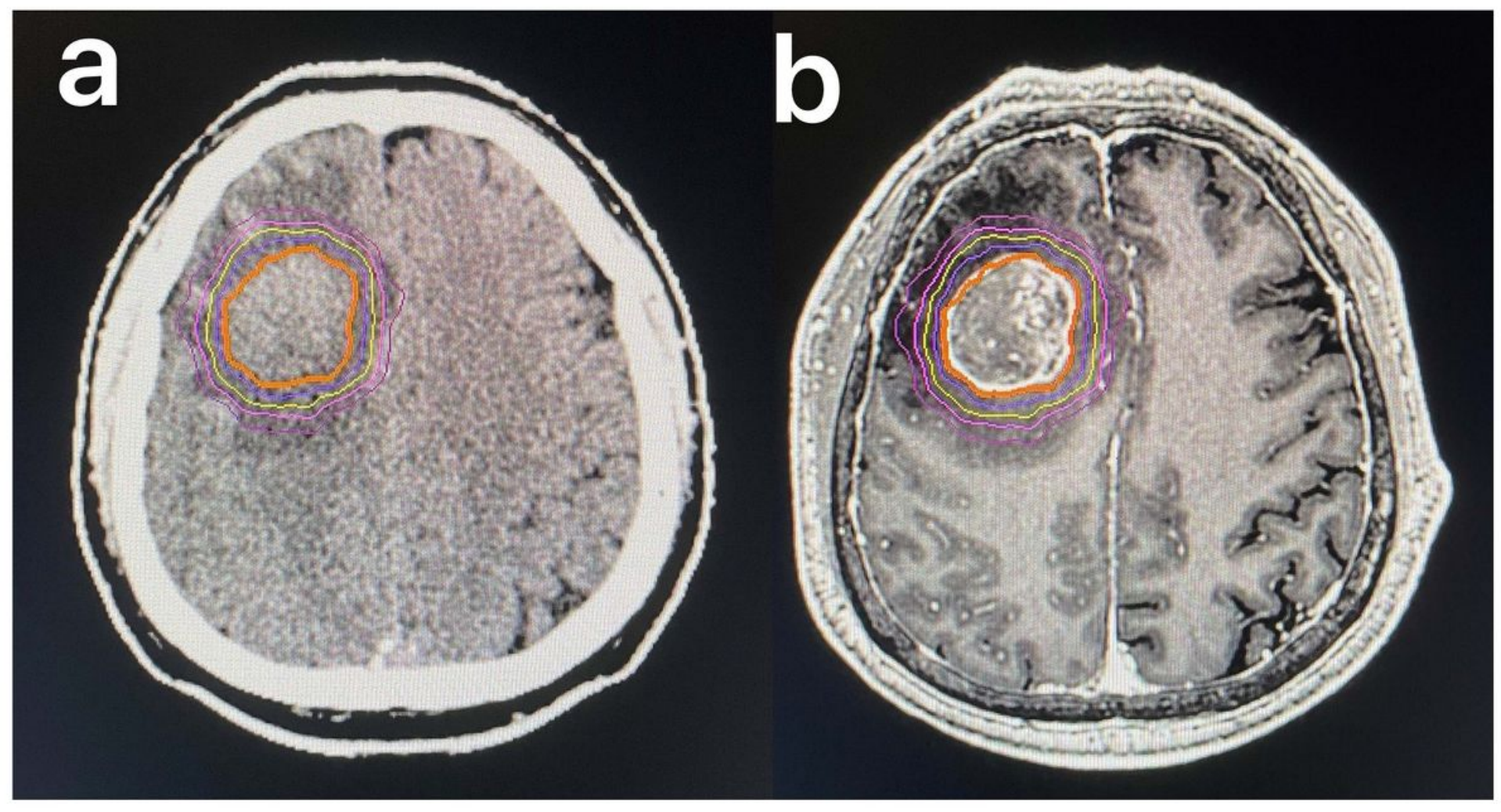

Figure 2

SBRT target area in a 58-year old male with squamous cell lung cancer of the right frontal lobe, CT (a) and MRI (b).

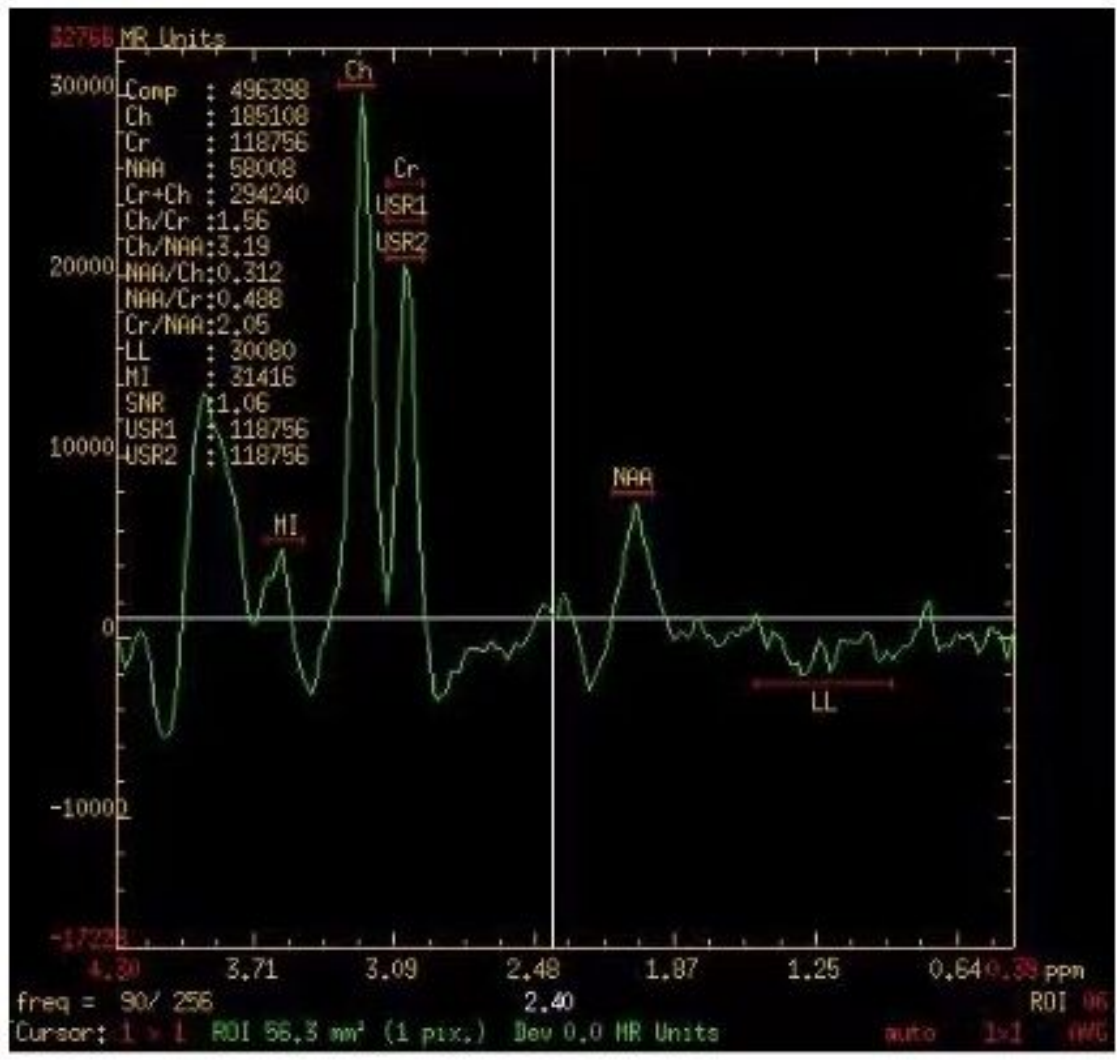


Figure 3

Magnetic resonance spectroscopy imaging of a 66-year-old female with NSCLC.
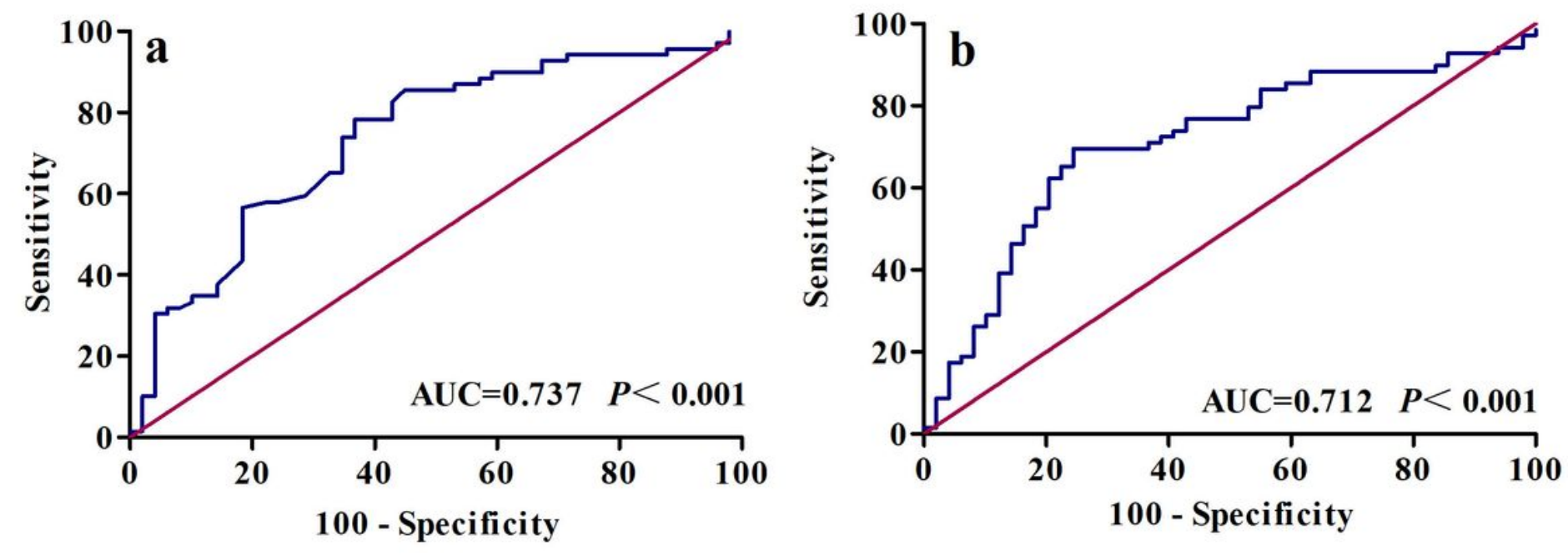

Figure 4

Receiver operating characteristic (ROC) curve analysis of the (a) Cho/Cr and (b) SII.

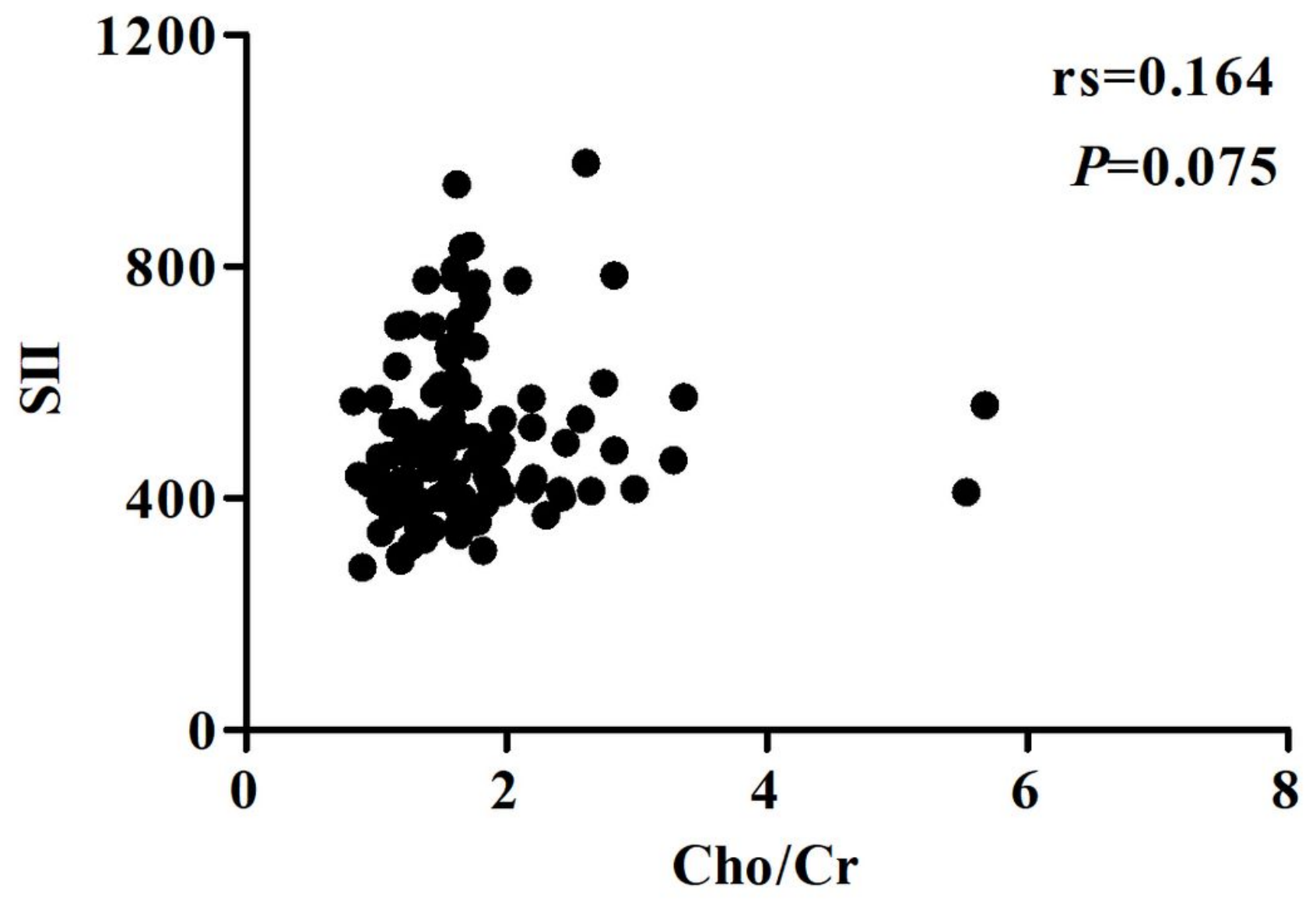


Figure 5

Correlations between Cho/Cr and SII.
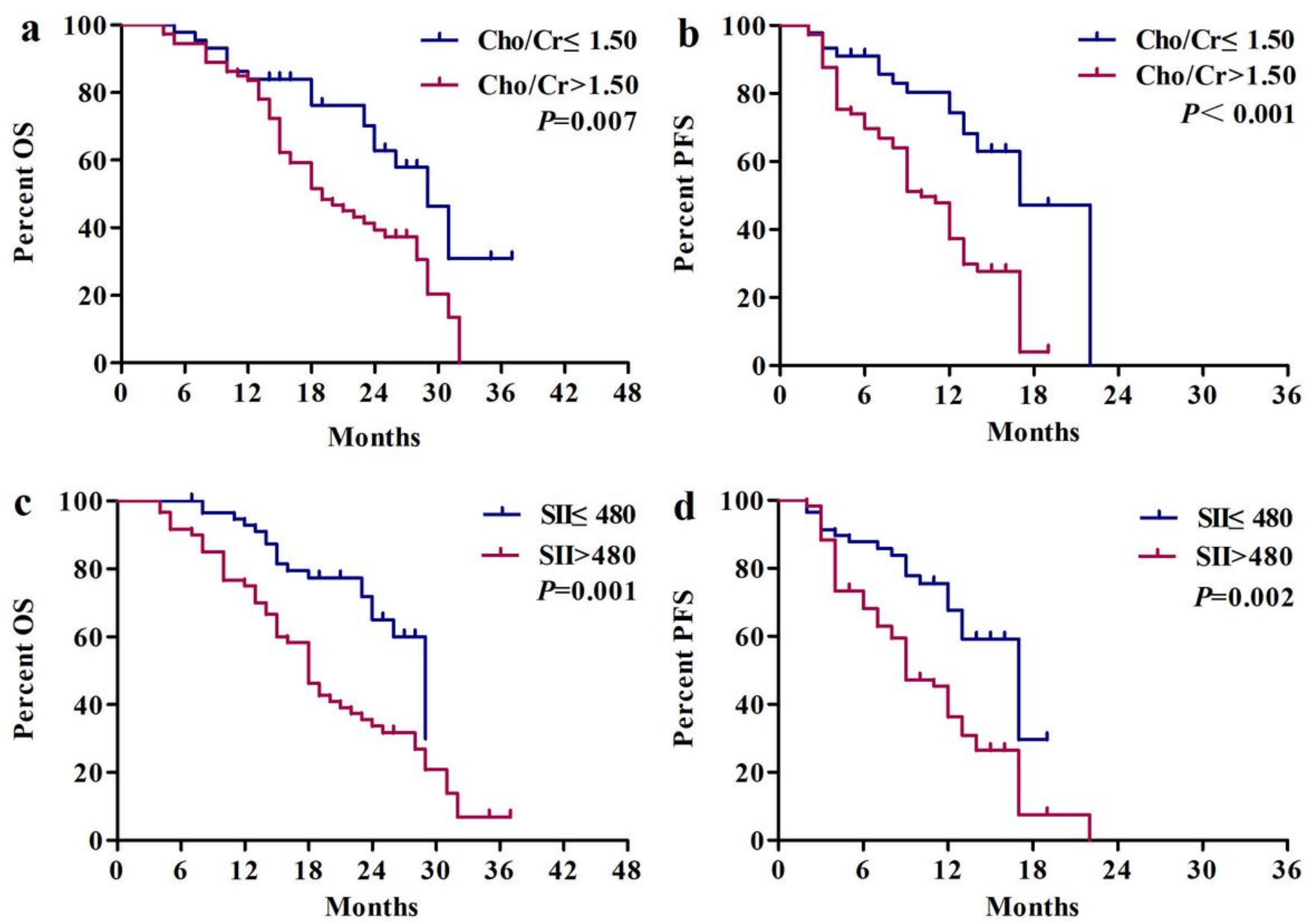

Figure 6

Kaplan-Meier survival curves of Cho/Cr and SII in NSCLC patients with brain metastases. a overall survival curves of Cho/Cr. b progression-free survival curves of SIl. c overall survival curves of $\mathrm{Cho} / \mathrm{Cr}$. $\mathrm{d}$ progression-free survival curves of SII 

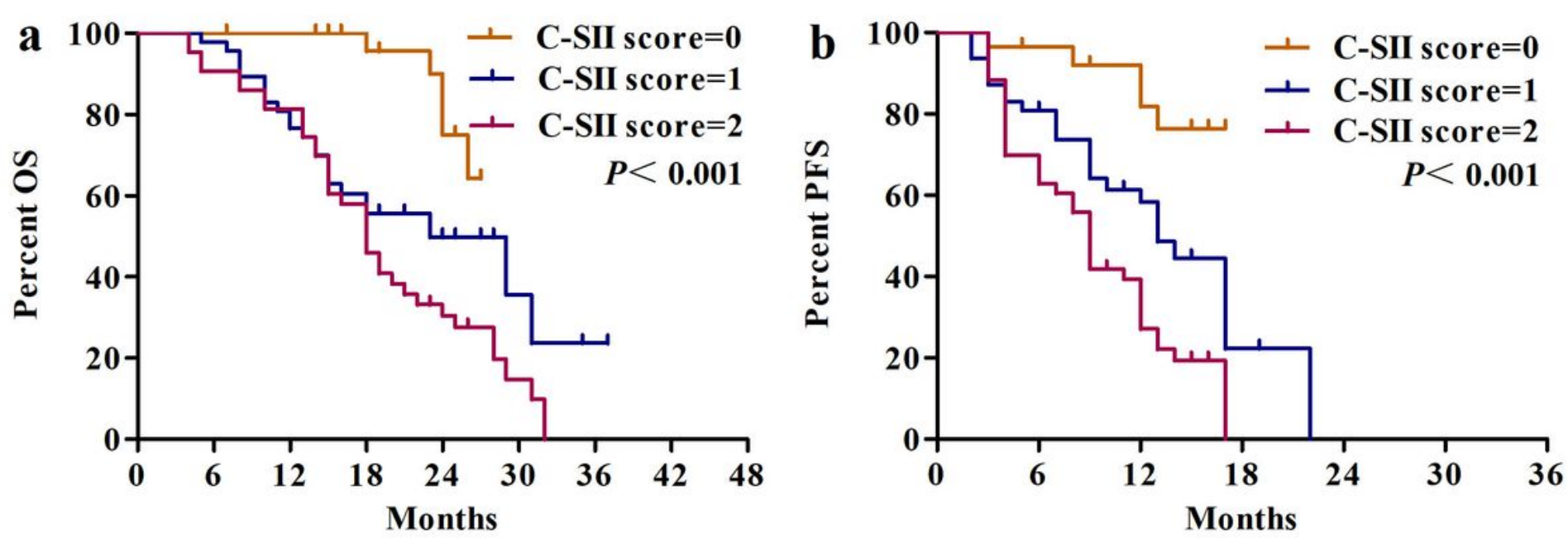

Figure 7

Kaplan-Meier survival curves depicting outcomes of overall survival (a) and progression-free survival (b) according to the C-SII score. 